\title{
ORIENTAÇÕES PARA PROFESSORES DE FÍSICA QUE SE AVENTURAM NA REALIZAÇÃO DE VIDEOANÁLISES UTILIZANDO O SOFTWARE
}

\author{
TRACKER \\ Emanuelly Torres Nunes ${ }^{1}$ \\ Ivanderson Pereira da Silva ${ }^{2}$
}

DOI: $10.29327 / 3860.12 .21-3$

\begin{abstract}
RESUMO: A videonálise como instrumento para estimular a modelagem científica utilizando o software Tracker como ferramenta proporciona atividades experimentais significativas com a participação crítica e ativa dos alunos no processo de construção do conhecimento. $\mathrm{O}$ presente trabalho apresenta conceitos sobre a videoanálise como exploração da modelagem científica, bem como orientações de utilização e análise de dados utilizando o software Tracker. A videoanálise constitui-se em tecnologia de grande potencial para incentivar os estudantes a terem um papel de protagonistas no processo de ensino aprendizagem, permitindo que os mesmos possam escolher o fenômeno da sua realidade e analisá-lo de forma crítica. O software Tracker é uma boa escolha para a realização da videoanálise, pois é livre, de fácil acesso e fornece automaticamente, depois das marcações no objeto a ser analisado, as posições e intervalos de tempo quadro a quadro, gerando tabela de dados e até três tipos de gráficos do mesmo movimento. Demonstra-se passo a passo de uma videoanálise de movimento na vertical e possíveis análises que podem ser feitas por meio dos gráficos construídos no software.
\end{abstract}

PALAVRAS-CHAVE: Tracker; Videoanálise; Ensino de Física.

ABSTRACT: Video analysis as a tool to stimulate scientific modeling using Tracker software as a tool provides significant experimental activities with the critical and active participation of students in the process of knowledge construction. This paper presents concepts about video analysis as exploration of scientific modeling, as well as guidelines for use and data analysis using Tracker software. Video analysis is a technology of great potential to encourage students to play a leading role in the process of teaching and learning, allowing them to choose the phenomenon of their reality and critically analyze it. Tracker software is a good choice for video analysis because it is free, easily accessible and automatically provides, after the markings on the object to be analyzed, the positions and time intervals frame by frame, generating data table and up to three chart types of the

\footnotetext{
${ }^{1}$ Professora de Física da Rede Pública Estadual de Educação de Alagoas. Mestranda em Ensino de Ciências e Matemática pelo Programa de Pós-graduação em Ensino de Ciências e Matemática da Universidade Federal de Alagoas; Licenciada em Física pela Universidade Federal de Alagoas.

${ }^{2}$ Professor Permanente do Programa de Pós-Graduação em Ensino de Ciências e Matemática da Universidade Federal de Alagoas. Mestre e Doutor em Educação pelo Programa de Pós-graduação em Educação da Universidade Federal de Alagoas.
} 
same motion. We will demonstrate step by step a vertical motion video analysis and possible analyzes that can be done through the graphics built in the software.

KEYWORDS: Tracker. Video Analysis. Physics Education.

\section{INTRODUÇÃO}

A experimentação apoiada em Tecnologias Digitais da Informação e Comunicação (TDIC) potencializa ganhos pedagógicos e tende a "oferecer a professores e estudantes ferramentas computacionais que possibilitem modos diferentes de descrever, explicar, prever e entender fenômenos físicos”. (BEZERRA JR. et al., 2012, p. 473). Dentre as TDIC disponíveis e que podem apoiar a experimentação em Física no contexto da Educação Básica, destacamos o software Tracker, disponível em: https://physlets.org/tracker/, que consiste em um programa gratuito e que possibilita metodologias de experimentação baseadas em videoanálise e aplicação do conhecimento da disciplina de Física de forma ativa e investigativa.

Nesse estudo, este ensaio tem por objetivo principal demonstrar instruções de utilização e manipulação das ferramentas do software Tracker. De modo específico, se objetiva detalhar as ferramentas do software Tracker; especificar passos para a realização de uma videoanálise; e apresentar tipos de gráficos que podem ser gerados na videoanálise pelo software.

O uso software Tracker permite desenvolver atividades centradas no processo de modelagem científica, através da videoanálise de movimentos, permitindo que os alunos acompanhem a evolução das grandezas físicas por meio de vídeos dos fenômenos reais. Tem uma função mediadora no ensino dos conteúdos de forma mais exata e rápida, sem necessidade de sensores e equipamentos de alto custo.

A experimentação tem um papel importante no processo formativo e a videoanálise, pode complementar a prática experimental em Física. Proporcionando, em sintonia com as TDIC, oportunidades aos alunos de observar a natureza, coletar e analisar dados, de forma ativa e crítica no processo de construção de conhecimento e compreensão da sua realidade.

Trata-se de um estudo descritivo (Sampieri; Collado; Lúcio, 2013) no qual dispomos, em um primeiro momento, uma descrição do processo de modelagem 
científica utilizando como ferramenta o software Tracker; na sequência apresentamos as funcionalidades e as potencialidades didáticas do software Tracker, e por fim discorremos sobre os tipos de gráficos que podem ser gerados bem como os conteúdos que podem ser estudos a partir da videoanálise.

\section{MODELAGEM CIENTÍFICA E SOFTWARE TRACKER}

A modelagem científica permite que os alunos possam compreender sua realidade utilizando um modelo computacional já construídos. Nesta seção, discutiremos a modelagem científica através da videoanálise utilizando o software Tracker como ferramenta.

Videoanálise é uma técnica que permite o registro de fenômenos que envolvam movimento por meio de filmagens que podem ser captadas por simples câmeras de celulares, e que podem ser utilizadas como atividades experimentais nas aulas de física, permitindo aos alunos o acompanhamento de grandezas físicas, manipulação dos dados, construção de gráficos e o papel de agentes ativos na construção do conhecimento.

Em atividades experimentais convencionais em laboratórios de física utilizam sensores e equipamentos para registrar dados, mas geralmente o registro do tempo é feito através de um cronômetro que precisa ser acionado com maior agilidade possível a fim de obter menos erros nas coletas de dados, pois essa aquisição de dados pode ser prejudicada pela rapidez do movimento. Já na videoanálise "o software fornece, automaticamente, os valores de distância a partir de um padrão e também identifica automaticamente a quantidade de quadros por segundo empregada pela câmera digital usada” (SAAVEDRA FILHO et al, 2017).@@@@@ @ ver pág. Da citação

Nesse sentido, a utilização de uma ferramenta de mediação tecnológica, como o software Tracker, pode levar a resultados mais precisos do que procedimentos manuais, além de permitir a evolução temporal nas grandezas através dos gráficos que automaticamente são construídos a medida que as posições são marcadas, como descreveremos na seção seguinte. 


\section{FUNCIONALIDADES E POTENCIALIDADES DIDÁTICAS DO SOFTWARE} TRACKER

O recurso da videoanálise pode favorecer uma aproximação com o fazer científico. Nesse sentido, o software Tracker se apresenta como uma ferramenta que viabiliza essa aproximação a partir de um computador de baixa configuração (BEZERRA JR et al., 2012). Segundo Saavendra Filho et al. (2017, p. 234)

O software Tracker permite aos alunos acompanharem a evolução das grandezas físicas, a manipulação dos dados e construção dos gráficos, além de proporcionar que os atores deste processo passem a ser agentes ativos na construção, customização e adequação do programa às suas realidades.

Segundo Lenz (2012, p. 18) “o Tracker adiciona qualidade e praticidade às aulas de Física, pois não são necessários aparatos experimentais caros, complexos e laboriosos com os quais, frequentemente, os estudantes 'perdem' um tempo precioso nas aulas de laboratório".

O Tracker permite a análise de vídeo e modelagem desenvolvido para o ensino de Física. Possibilita o rastreamento da posição de um objeto, gráficos e dados de velocidade e aceleração de corpos em movimento, calibração de medidas, divisão do vídeo quadro a quadro, rotação de posição do vídeo, indicação de grandezas vetoriais, trajetórias e coordenadas.

Entende-se que o uso do Tracker esteja em sintonia com a modernização do saber escolar, em um contexto em que as atividades experimentais teriam função mediadora no ensino dos conteúdos (BEZERRA JR et al., 2012). O software Tracker possibilita o desenvolvimento de uma aprendizagem significativa, oferecendo aos professores e alunos metodologias de experimentação e aplicação do conhecimento da disciplina de Física de forma ativa e investigativa, desenvolvendo a criatividade e o pensamento crítico. Como afirma Saavendra Filho et al. (2017, p.242)

O uso do Tracker no ensino de Física e Engenharia é promissor por conta de seu baixo custo, de sua versatilidade e do interesse que desperta nos estudantes, tendo em vista a dinâmica de aulas em que permite a participação ativa dos estudantes e em uma posição em que os questionamentos decorrentes da metodologia proposta permitam formular, questionar, predizer, testar hipóteses- passos importantes em um processo de modelagem científica. 
A utilização do programa Tracker como ferramenta possibilita minimizar a ausência de laboratórios de ciências, e possibilita o contato com as TDIC para realizar análises de observações de fenômenos físicos, mais especificamente do movimento, presentes no cotidiano dos alunos.

O software por si só não é suficiente. Faz-se necessário que a instituição tenha estrutura de laboratório de informática e acesso à internet, como ressaltam Bezerra Jr. et al. (2018). Entendemos que a inclusão digital depende não apenas do acesso à formação básica em software, mas também necessite da ampliação do acesso à internet e também ao computador.

O Tracker pode ser obtido no site https://physlets.org/tracker/ disponível para os sistemas operacionais Windows, Mac OS X, Linux 32-bit e Linux 64-bit. É um software de fácil aprendizagem que pode ser manuseado pelos alunos em experimentos didáticos de Física.

Com o software instalado no computador é possível o seu manuseio sem necessidade de acesso à internet. Ao abrir o software a tela inicial terá a mesma aparência da figura 1, onde podemos observar a barra de ferramenta, parte central onde será projetado o vídeo e, na direita da tela, local onde serão apresentados os gráficos e tabelas. 
Figura 1- Tela Inicial

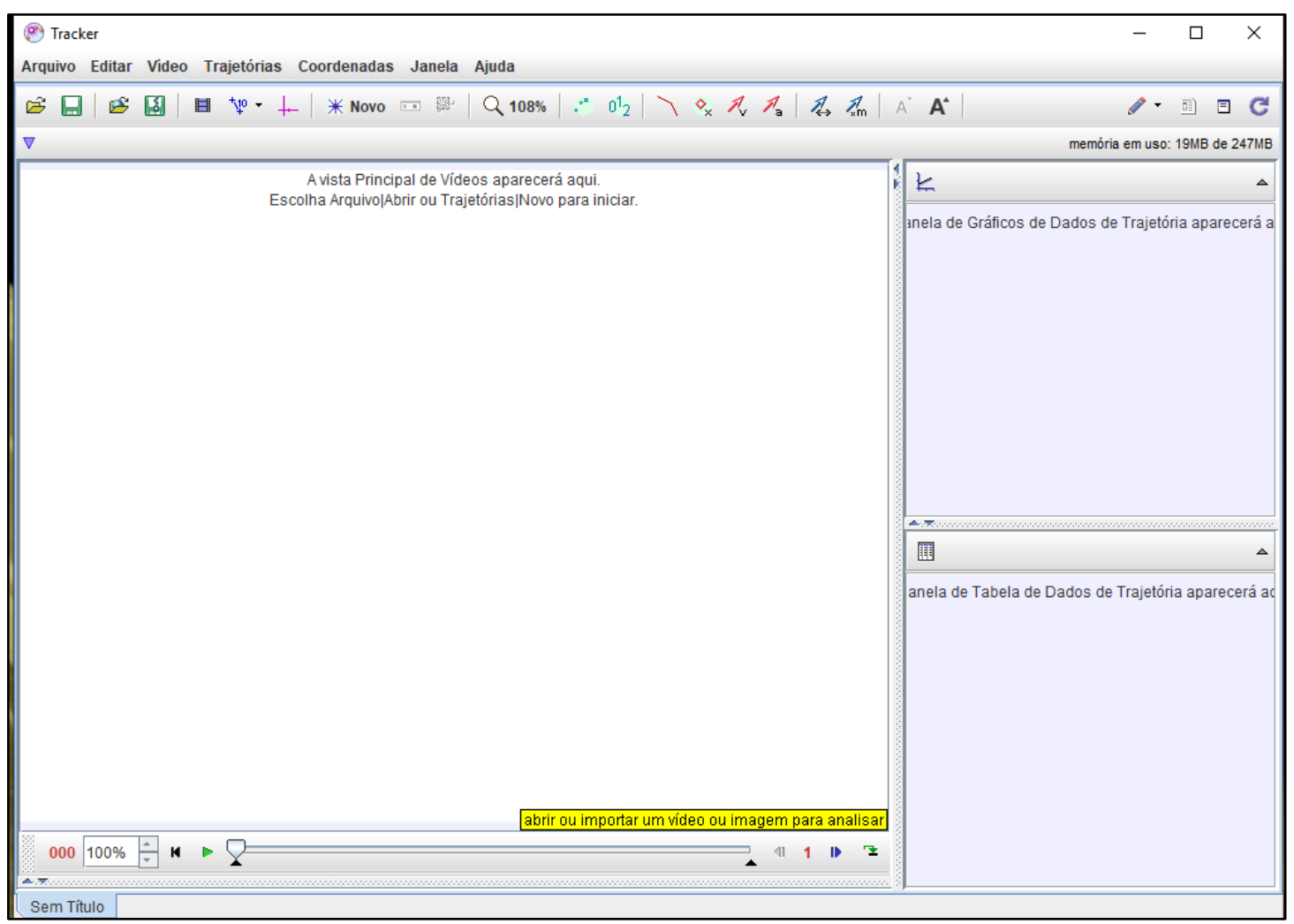

Para iniciar a videoanálise é necessário importar o vídeo através da barra de ferramenta (Figura 2), que deverá estar salvo em alguma pasta do computador que facilmente possa ser encontrado, como mostra na figura 3. 
Figura 2 - Importar Vídeo

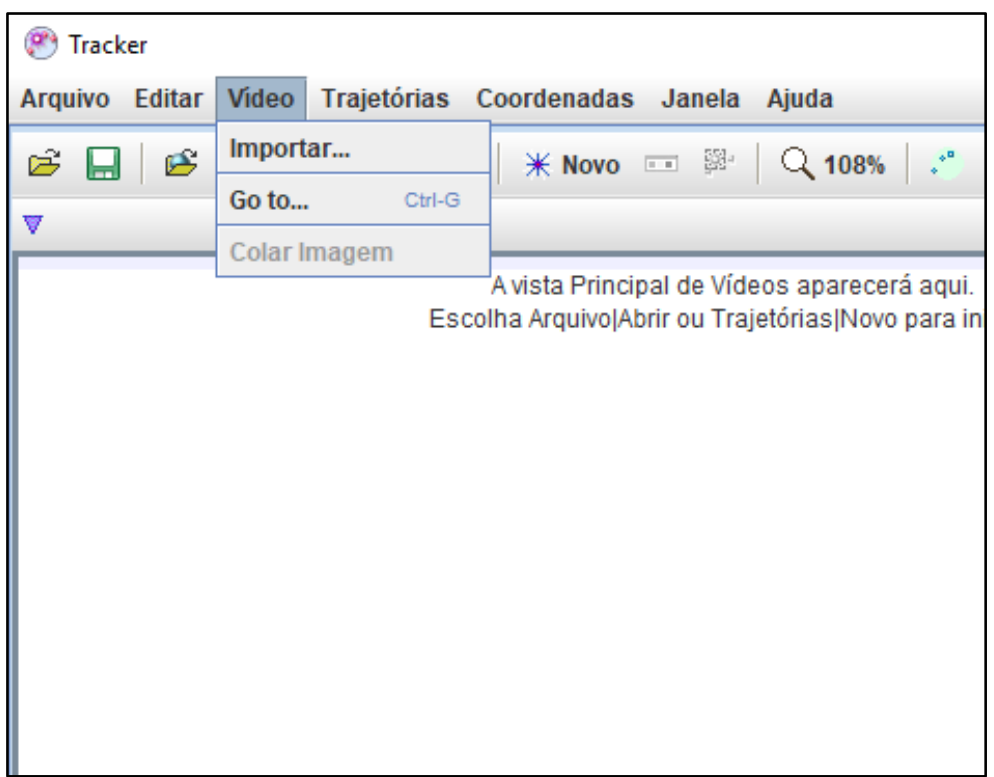

Figura 3 - Escolher vídeo no computador

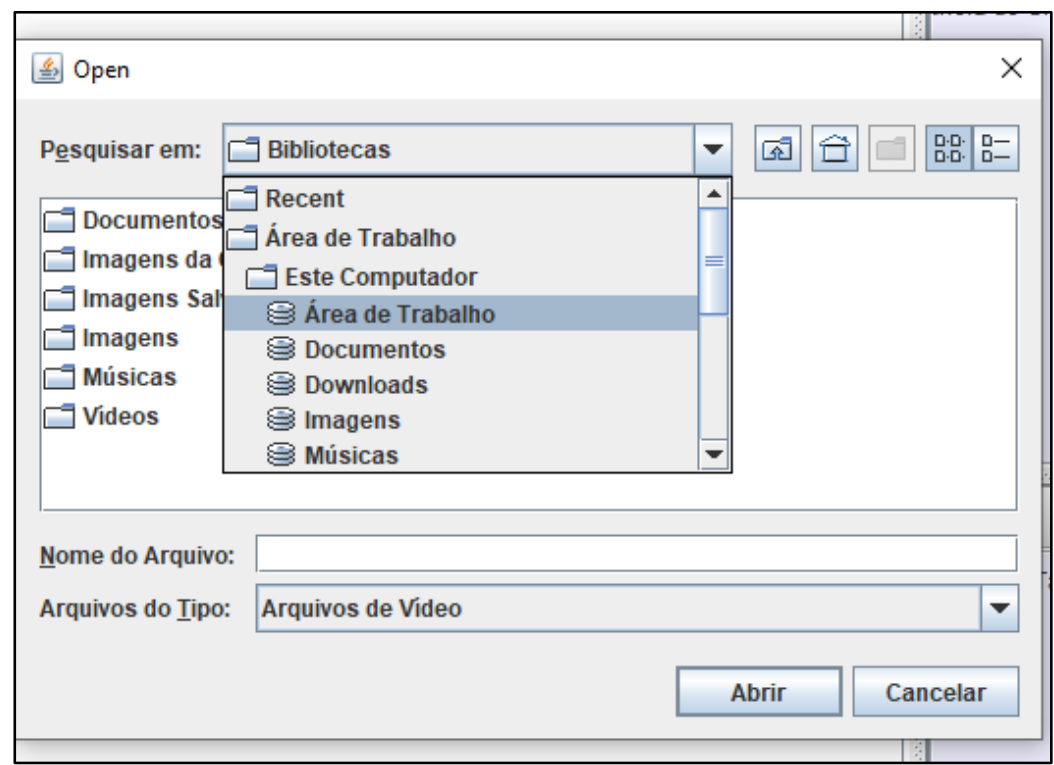

Se for preciso girar o vídeo para outra posição é necessário seguir os seguintes comandos na barra de ferramenta vídeo $>$ filtros $>$ novo $>$ rotacionar, como mostra a figura 4, e aparecerá a janela semelhante à da figura 5. Assim será possível girar o vídeo na posição desejada. 
Figura 4 - Modificar a posição do vídeo

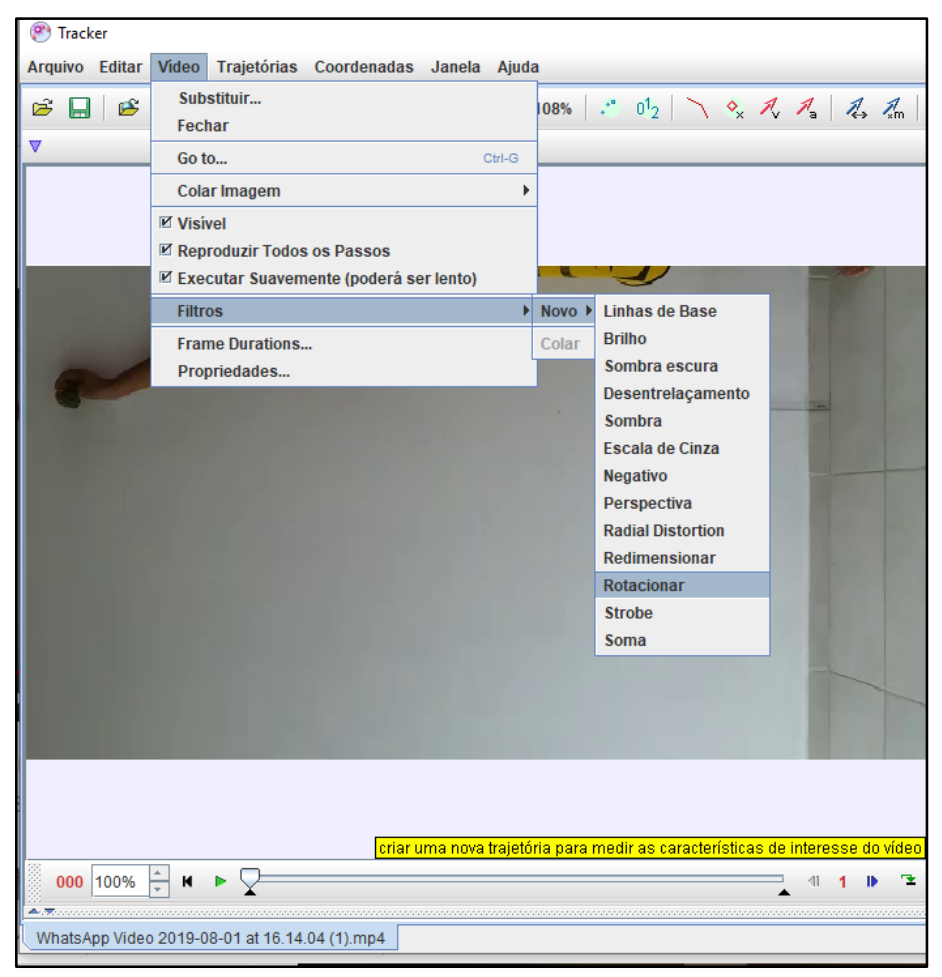

Figura 5 - Opção de rotação

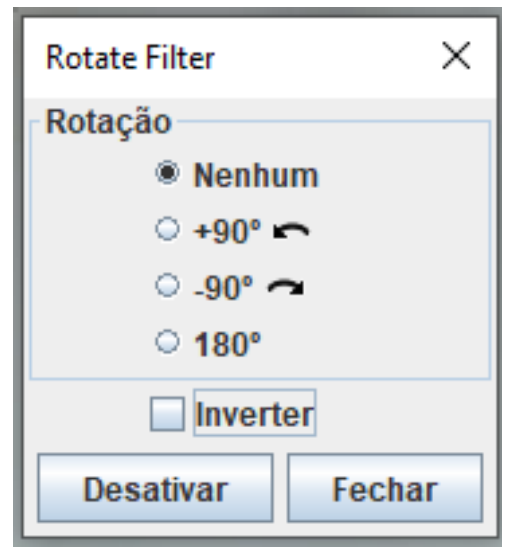

É aconselhável realizar o recorte do vídeo da situação que será analisada. Esses ajustes podem ser definidos na barra inferior da tela como estão sinalizados na figura 6 . Logo depois podemos salvar o recorte do vídeo através da barra de ferramenta como mostra a figura 7 , e abri-lo logo em seguida para iniciar a análise detalhada do movimento.

Figura 6 - Barra de ajuste do intervalo do vídeo 


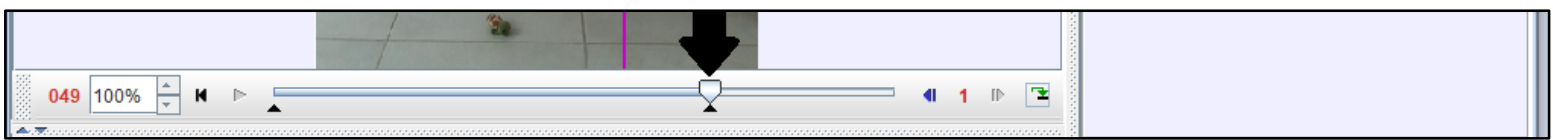

Figura 7 - Salvar recorte do vídeo

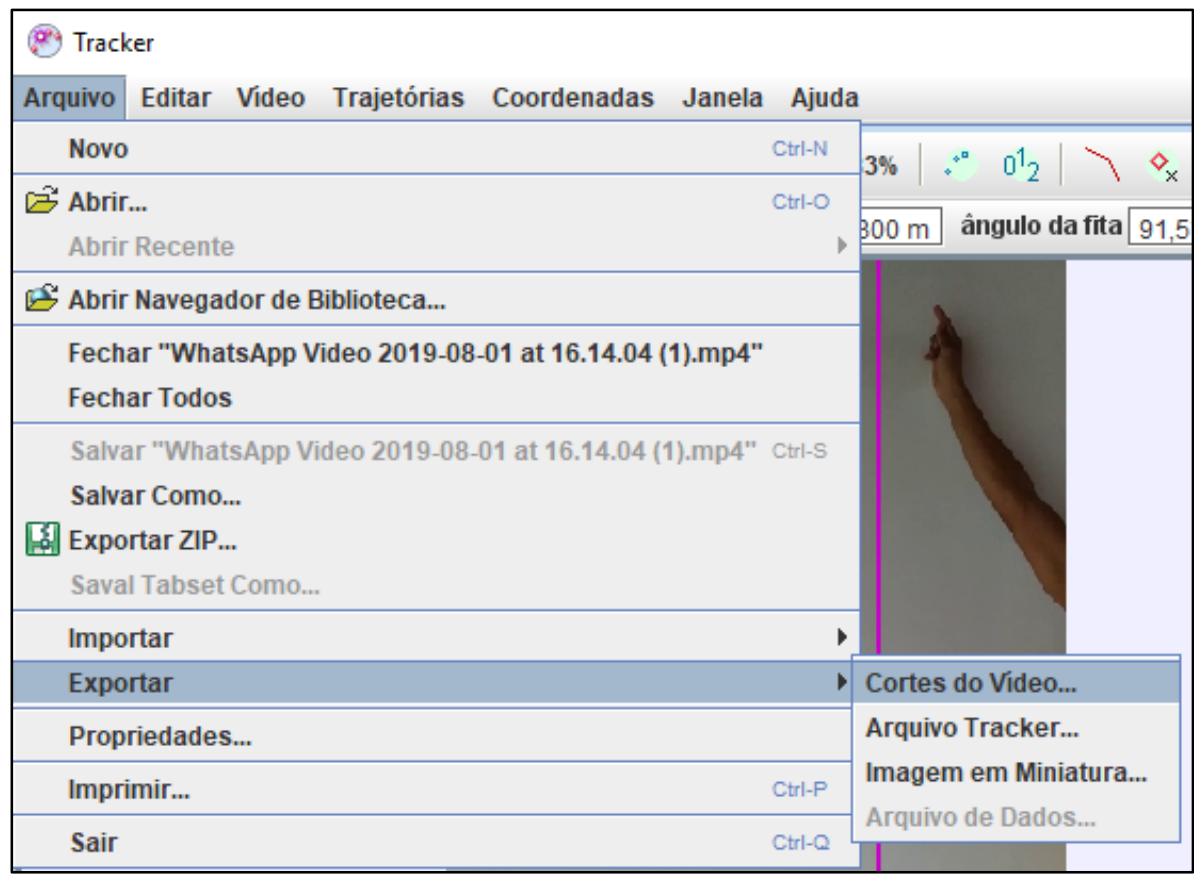

Para iniciar a videoanálise situaremos, por meio através da barra de ferramentas, o eixo de coordenadas e uma fita de calibração, Propositalmente foi colocada uma régua de $30 \mathrm{~cm}$ no local da gravação, calibrando assim a escala do vídeo. Como podemos visualizar nas figuras 8 e 9 .

Figura 8 - Fita de calibração

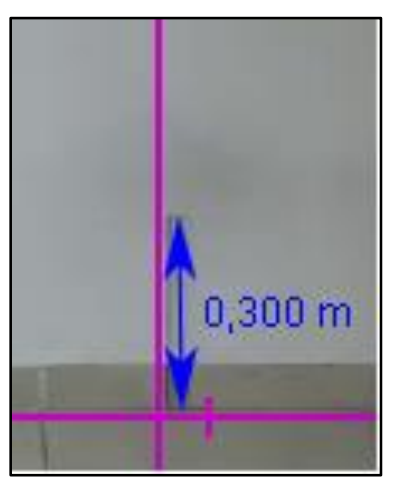


Figura 9 - Eixo de Coordenadas

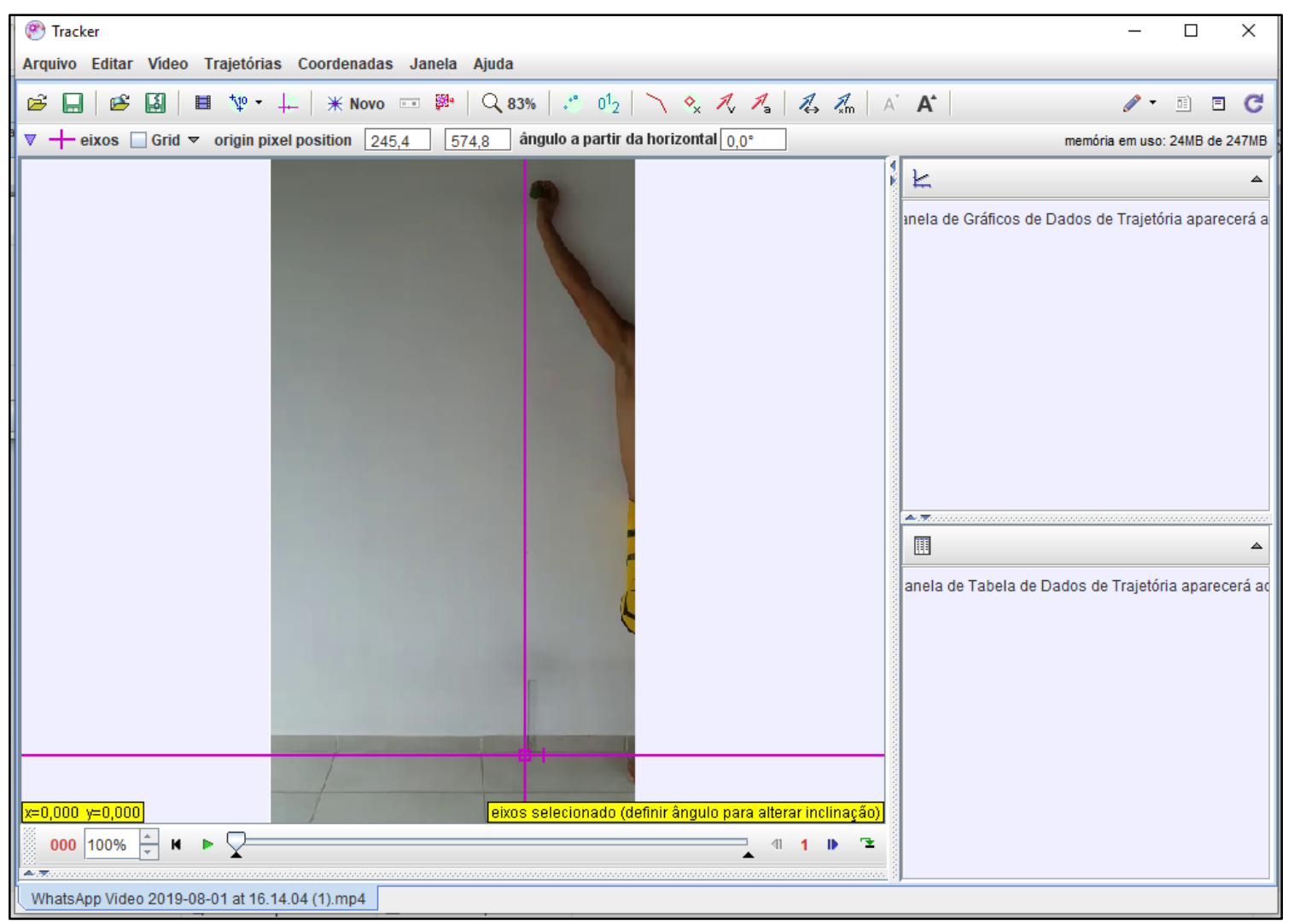

Para marcar o objeto que terá o seu movimento analisado é necessário ir em Novo, localizado na barra de ferramenta, e escolher Ponto de Massa (Figura 10). Em seguida, marcar trajetória indo em Massa A > Marcar como Padrão (Figura 11) e clicar no centro do objeto a cada avanço de quadro na barra de ajuste, marcando assim as posições quadro a quadro, que sugiram nos gráficos e tabela. 
Figura 8 - Marcar objeto

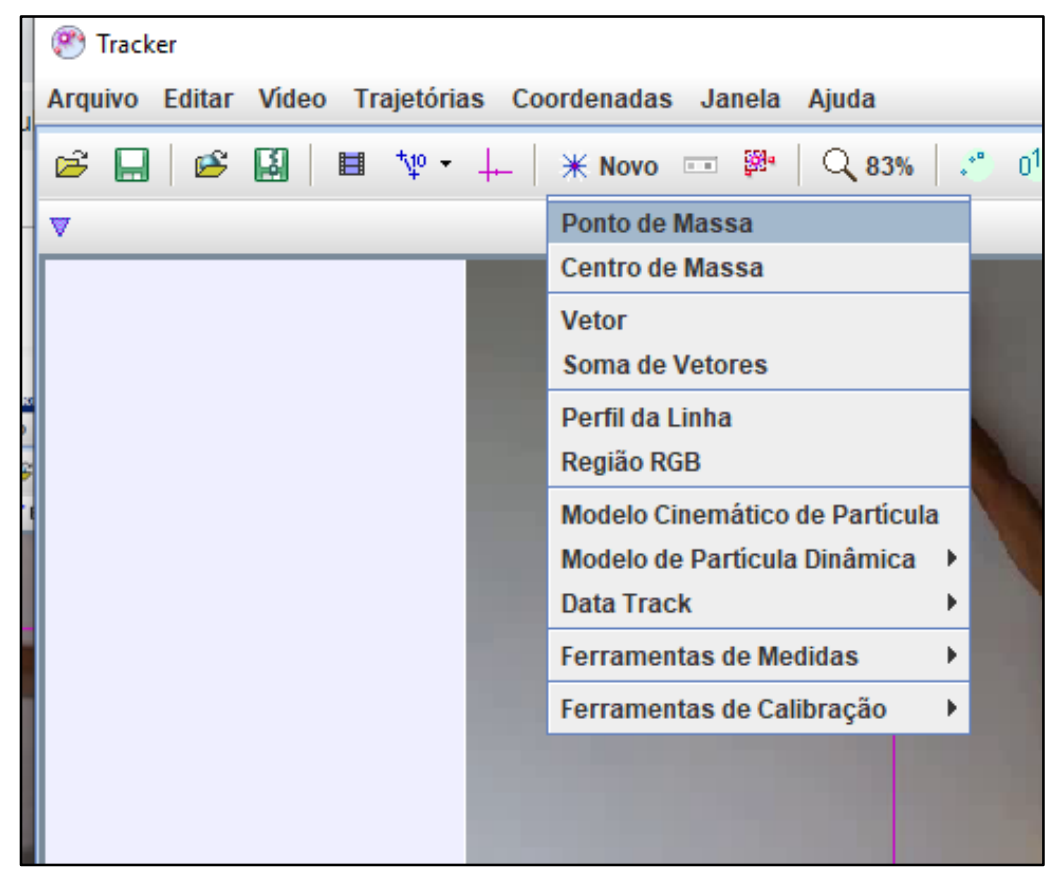

Figura 9 - Marcar objeto quadro a quadro

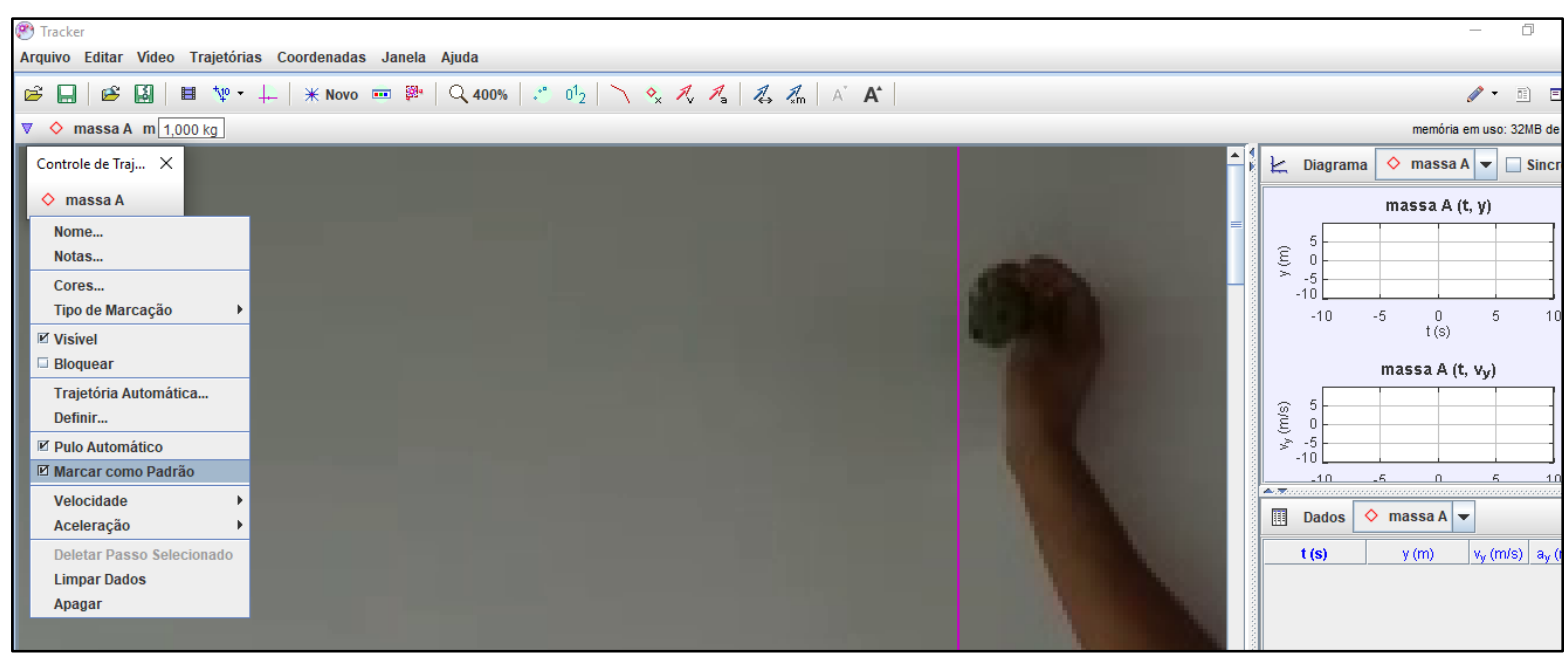

Na parte direita da tela do software, onde serão apresentados os gráficos, é possível escolher a projeção de um a três gráficos (diagramas). Podendo também ser modificadas as grandezas físicas que serão apresentadas no gráfico. Na parte inferior desse mesmo lado é possível escolher as grandezas que serão analisadas e apresentadas na tabela. Nesse caso será exibida uma tela semelhante à da figura 12 . 
Figura 10 - Escolha das grandezas que serão apresentadas na tabela

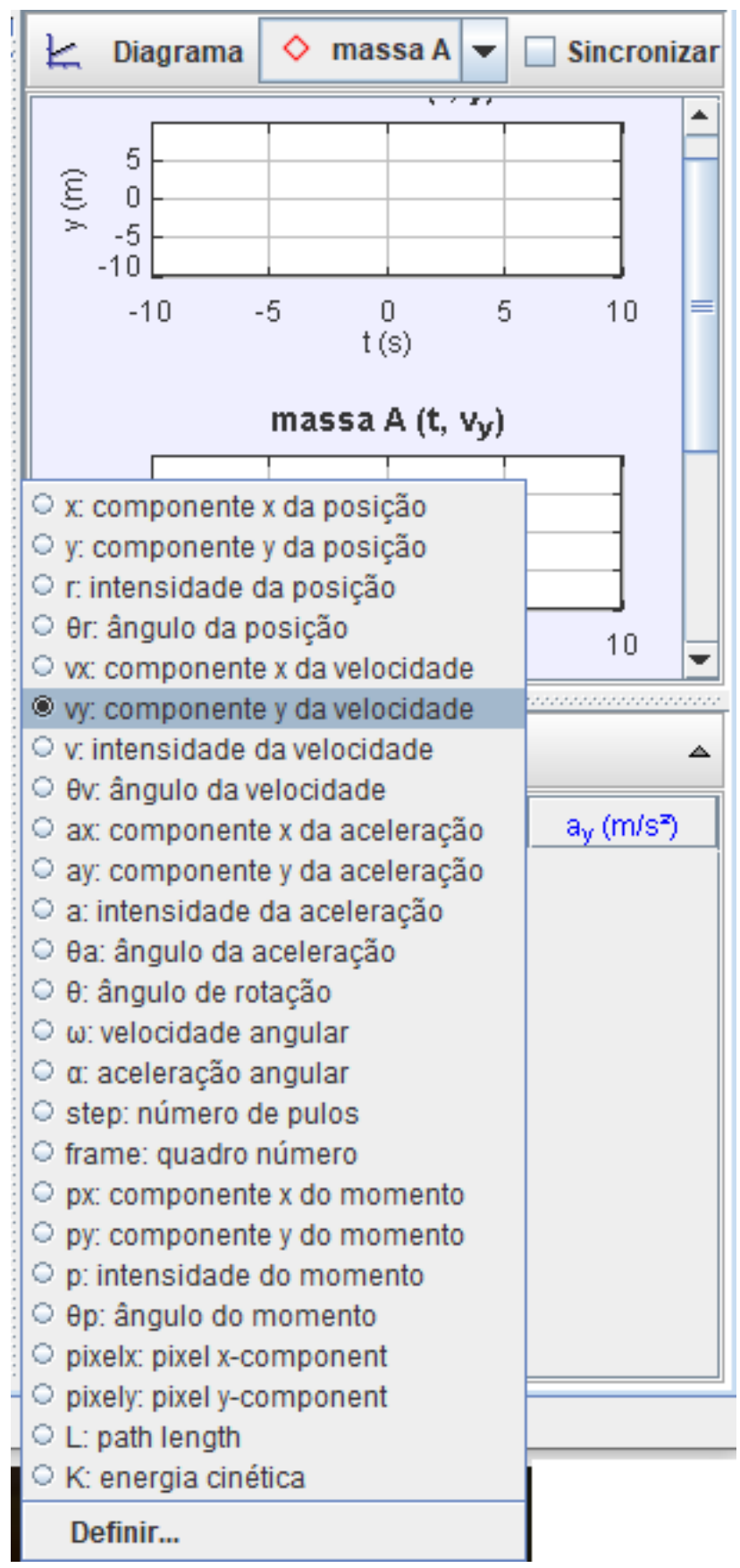

Depois de marcar as posições do objeto, quadro a quadro, novas informações serão apresentadas no lado direito da tela. Essas poderão ser analisadas e discutidas de forma ativa e crítica pelos alunos. 
Figura 11- Posição do objeto quadro a quadro

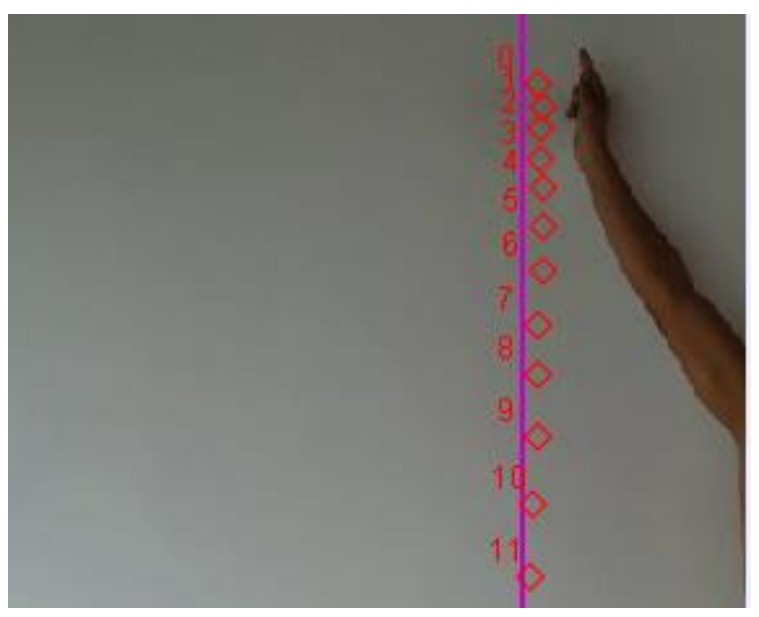

Ao clicar no objeto durante o movimento estaremos identificando sua posição naquele determinando tempo, podendo no final observar as variações das posições, quadro a quadro, como mostra a figura 13. Esta imagem apresenta as posições, quadro a quadro, de um movimento de queda livre, onde existe a aceleração da gravidade que pode ser calculada através dos dados obtidos pelas tabelas e gráficos. Mas o software Tracker também pode ser utilizado para estudos de diferentes de movimento.

Na figura 14 podemos observar três diagramas do estudo do movimento na posição vertical versus o tempo, velocidade vertical versus o tempo e aceleração vertical versus o tempo, que serão preenchidos à medida que os pontos são marcados quadro a quadro. Além dos gráficos são apresentados os dados nas tabelas.

Figura 12: Gráficos e Tabelas 


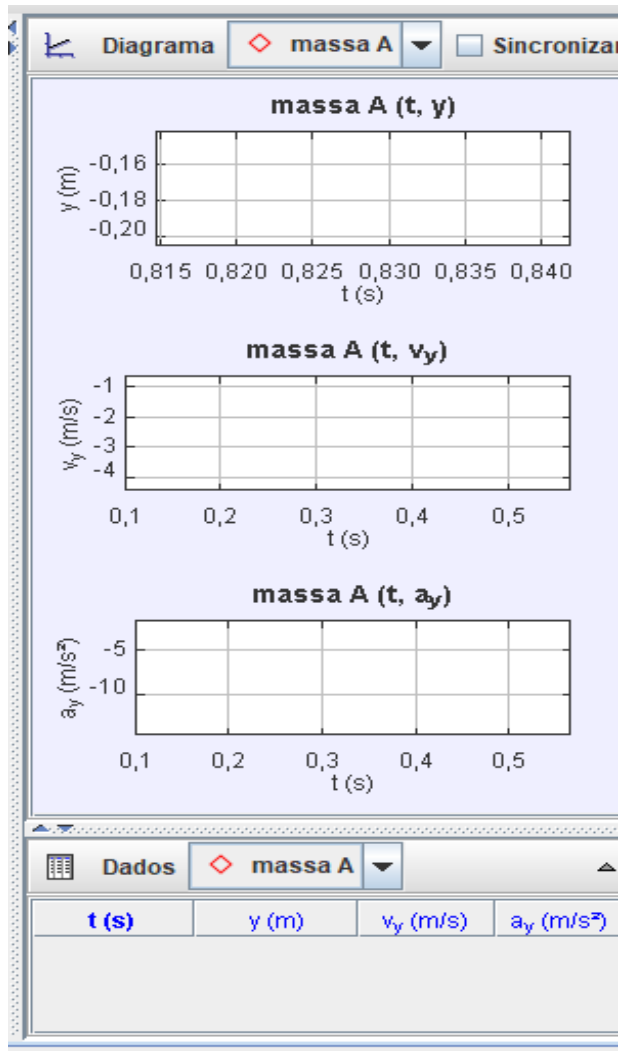

O software permite que sejam construídos de 1 a 3 gráficos (diagramas) que podem ser modificados. As grandezas que serão registrada nos eixos, e o zoom pode ser modificado ao clicar-se o botão direito do mouse.

\section{CONSIDERAÇÕES FINAIS}

O professor ao utilizar o software Tracker ou as TDIC de modo geral, deve promover atividades que ampliem as possibilidades de aprendizagem de seus alunos; estimulem sua curiosidade, trabalhando o pensamento crítico dos alunos, a fim de que eles possam ter um papel ativo em seus percursos de aprendizagem.

O uso do software Tracker como ferramenta para execução da videoanálise em atividades de modelagem científica, permite quebras de rotinas no contexto escolar, proporcionando uma participação crítica e ativa dos alunos na realização de experimentos significativos de vídeos de fenômenos do cotidiano, obtenção de medidas mais 
confiáveis, bem como despertar nos estudantes para o uso consciente da tecnologia e a importância da sua apropriação.

\section{REFERÊNCIAS}

BEZERRA JR, Arandi Ginane et. al. Atividades experimentais de física mediadas por videoanálise e o software livre Tracker na formação inicial de professores. In: ENPEC, 2017, Florianópolis. Anais [...]Encontro Nacional de Pesquisa em Ensino de Ciências, 11, 2017, Florianópolis. Disponível em: http://www.abrapecnet.org.br/enpec/xienpec/anais/listaresumos.htm. Acesso em: 22 de ou TESTAR ESSE LINKt.2018.

BEZERRA JR, Arandi Ginane et. al. Videoanálise com o software livre Tracker no laboratório didático de Física: movimento parabólico e segunda Lei de Newton. Caderno Brasileiro de Ensino de Física, v.29, n. Especial1: p.469-490, set. 2012.

BEZERRA JR, Arandi Ginane; LENZ, Jorge Alberto; SAAVEDRA FILHO, Nestor Cortez. Utilização da videoanálise para o estudo do movimento circular e a construção do conceito de aceleração centrípeta. Revista Acta Scientiae, Canoas, v.18, n.3, p.782798, set./dez. 2016.

CARVALHO, Ana Maria P. de (Org..). Ensino de ciências por investigação: condições para implementação em sala de aula. São Paulo: Cengace Learning, 2013.

FIOLHAIS, C.; PAIVA, J. Aristóteles, Galileu e a queda dos graves. Gazeta de Física, Lisboa, v. 15, p. 28-33, 1992.

LENZ, Jorge Alberto; SAAVERDRA FILHO, Nestor Cortez; BEZERRA JR, Arandi Ginane. Utilização de TIC para o estudo do movimento: alguns experimentos didáticos com o software Tracker. Revista ABAKÓS, Belo Horizonte, v. 2, n. 2, p.24-34, maio, 2014.

MENEGOTTTO, José Carlos; ROCHA FILHO, João Bernardes da. Atitudes de estudantes do ensino médio em relação à disciplina de Física. Revista Electrónica de Enseñanza de las Ciencias, v. 7, n. 2, 2008. VER DE ONDE É ESTA REVISTA.

SAAVENDRA FILHO, Nestor Cortez et al. A videoanálise como mediadora da modelagem científica no Ensino de Mecânica. Revista Brasileira de Ensino de Ciências e Tecnologia, Ponta Grossa, v. 10, n. 3, p. 231-246, set/dez, 2017.

SANTOS, Bianca M. et al. Relato de experiência: O uso de experimentos e mnemônicos no ensino de circuitos elétricos para alunos da zona rural. Educação Básica Revista, vol. 4, n.2, 2018.

ZABALA, Antoni. A prática educativa: como ensinar. Porto Alegre: ArtMed, 1998. 


\section{Emanuelly Torres Nunes}

Professora de Física da Rede Pública Estadual de Educação de Alagoas. Mestranda em Ensino de Ciências e Matemática pelo Programa de Pós-graduação em Ensino de Ciências e Matemática da Universidade Federal de Alagoas; Licenciada em Física pela Universidade Federal de Alagoas

\section{Ivanderson Pereira da Silva}

Professor Permanente do Programa de Pós-Graduação em Ensino de Ciências e Matemática da Universidade Federal de Alagoas. Mestre e Doutor em Educação pelo Programa de Pós-graduação em Educação da Universidade Federal de Alagoas.

Artigo recebido em 07/09/2019

Aceito para publicação em 29/01/2020

\section{Para citar este trabalho:}

NUNES, Emanuelly Torres; SILVA, Ivanderson Pereira da. ORIENTAÇÕES PARA PROFESSORES DE FÍSICA QUE SE AVENTURAM NA REALIZAÇÃO DE VIDEOANÁLISES UTILIZANDO O SOFTWARE TRACKER. Revista Paidéi@. Unimes

Virtual. . Volume 12 - Número 21. Janeiro 2020. Disponível em:

http://periodicos.unimesvirtual.com.br/index.php/paideia/index 